\title{
Planar Leaky Light-Guides and Couplers
}

\author{
R. Ulrich and W. Prettl \\ Max-Planck-Institut für Festkörperforschung, Stuttgart, F.R. Germany
}

Received 21 August 1972/Revised 21 September 1972

\begin{abstract}
A quantitative theory of light propagation in a dielectric slab guide with general cladding media is presented. It is based on a plane wave which bounces in a zigzag fashion along the guide as a result of total or partial reflections at the two surfaces of the film. Two mechanisms are considered which contribute to the attenuation of the guide: losses due to absorption in the slab and cladding materials, and radiation losses if the guide is a leaky one. We point out the significance of the Goos-Hänchen effect for all questions relating to the power flow in the slab guide. The theory is illustrated by discussing dispersion and attenuation of guides with various low-index and high-index claddings, operating above and below cutoff. The low-index leaky guide is considered particularly in detail. Its high attenuation by leakage can be reduced to practically acceptable values $(<1 \mathrm{~dB} / \mathrm{cm})$ by increasing the film thickness to $\gtrsim 40 \lambda$. One application of this guide is in the leaky wave coupler. This coupler may be viewed as a prism-film coupler simplified by omission of the gap. It offers a new approach to the problem of broad-band coupling to thin-film light guides.
\end{abstract}

Index Headings: Integrated optics - Thin films - Goos-Hänchen effect

The planar dielectric slab guide has become a model light guide for many experimental and theoretical studies relating to integrated optics [1-5]. It consists of a thin transparent, high-index film, sandwiched between two transparent low-index claddings. Propagation of light in this guide can be explained by assuming that a plane wave in the film undergoes total reflections alternatingly at the upper and lower surfaces of the film, and thus bouncing in a zigzag fashion along the guide $[2,5]$ (Fig. 1). This "classical" guide is a special case of a more general class of slab guides in which the relative magnitudes of the indices and of the losses in the three regions are all arbitrary. A discussion of this general class of guides is the subject of the present paper.

The existence of freely propagating modes is not restricted to guides based on total reflection of the wave at the interfaces between the slab and the claddings. Partial reflection can produce guided modes, too. However, their propagation along the guide is attenuated. Although this is basically undesirable, such guides are of interest because their attenuation can be reduced to practically acceptable levels by sufficiently increasing the thickness of the film. A reasonable discrimination against higher modes is also possible in such "thick-film" guides because their higher modes have considerably higher attenuations. Two reasons for nontotal reflections will be considered here: absorption of light in the cladding materials, and refraction of light into the claddings. In the former case the guide is lossy; in the latter case it is leaky.

A type of leaky guide that we shall discuss particularly is the planar equivalent of the hollow optical fiber: a low-index dielectric film sandwiched between two high-index claddings. The theoretical results on the attenuation of this guide have been confirmed experimentally. Other types of leaky guides are known in different contexts, e. g. as Lummer-Gehrcke plate or as prism-film coupler. Here they will be considered from the viewpoint of light-guiding. The low-index gap in the prism-film coupler, though advantageous for very thin guides, is not an absolute necessity. A gapless coupler can give the same 
coupling efficiency, provided the film thickness can be adjusted to a suitable thick value (typically $3 \mu \mathrm{m}$ ). Gapless couplers may be useful for measuring the attenuation of low-loss guides.

\section{Freely Propagating Modes in a Slab}

For a quantitative description of light-guiding in a slab we make the following rather general assumptions: the slab of thickness $W$ consists of a homogeneous material 1 of complex refractive index $\tilde{n}_{1}=n_{1}+i \kappa_{1}$. The "cladding" regions 0 and 2 , adjacent to the slab (Fig. 1), are homogeneous in the plane parallel to the slab, but they may be of arbitrary composition in the normal direction within an interval $|z| \leqq D$ of an arbitrary, but finite width $2 D$, containing the slab. Outside this interval, the cladding regions must be homogeneous also in the normal direction. We characterize the regions 0 and 2 in the following only by their amplitude reflection coefficients $r_{10}$ and $r_{12}$ at the boundaries to material 1 . When we discuss specific types of slab guides, we have to insert specific functions for $r_{10}$ and $r_{12}$, depending on the nature of regions 0 and 2 .

Let us take the mean direction of propagation as the $x$ axis of our coordinate system. The $z$ axis is normal to the slab. We consider only waves that are independent of the $y$ direction, i.e. $\partial / \partial y=0$.

In the interior of the slab, the field is the superposition of two plane waves $A$ and $B$ (see Fig. 1). Their spatial amplitude distribution is written as

$$
\begin{aligned}
V(x, z)= & A \exp \{i k(\beta x+\xi z)\} \\
& +B \exp \{i k(\beta x-\xi z)\} .
\end{aligned}
$$

From the symmetry of the system it follows that we have two polarization eigenstates, $T E$ and $T M$. In both cases, the amplitudes $A, B$, and $V$ denote the field component transverse to the $x z$ plane, which is the electrical field $E=E_{y}$ for $T E$ waves, or the magnetic field $H=H_{y}$ for $T M$ polarized waves. The remaining field components can then be derived by Maxwell's equations from $V(x, z)$. A time dependence $\exp (-i \omega t)$ of all fields with a constant frequency $\omega$ is tacitly assumed throughout this paper. By $k=\omega / c$ we denote the propagation constant of light in free space, so that $\beta$ and $\xi=\left(n_{1}^{2}-\beta^{2}\right)^{1 / 2}$ are the relative propagation constants along the slab and perpendicular to it, respectively. As $\beta$ is common to waves $A$ and $B$ and also [6] to the fields in regions 0 and 2, it describes the propagation of the entire field along the guide. Therefore, $\beta$ is the principal quantity characterizing the propagation along the guide. We allow for attenuation of the guided wave in the direction of propagation so that $\beta$ may be complex

$\beta=N+i K$.

Consequently, the waves $A$ and $B$ have plane phase fronts, but their amplitudes decay in $x$ direction. The real part $N$ of $\beta$ is related by $v_{\mathrm{ph}}=c / N$ to the phase velocity along the guide, i.e. $N$ is the effective refractive index $[4,7]$ of the guide. The imaginary part $K$ is the attenuation constant of the wave amplitude. The power decays as $I(x) / I(0)=\exp (-2 k K x)$, and the attenuation constant $\alpha$ in practical units is given by $\alpha=8.686 \mathrm{kK}$ decibels per unit length. Our goal now is to find the possible values of $N$ and $K$.

We obtain a condition for the free propagation of light along the slab guide from the requirement that, after completing one zigzag, the plane wave $\left(A^{\prime}\right.$ in Fig. 1) must be identical with the original wave ( $A$ in Fig. 1) so that the field is a unique function of space. We write $A^{\prime}=F A$, where the factor $F$ accounts for the reflections at the two film surfaces and for two traversals of the film thickness. We find the condition

$$
\begin{aligned}
F\left(\beta, \tilde{n}_{1}\right) \equiv & r_{10}\left(\beta, \tilde{n}_{1}\right) r_{12}\left(\beta, \tilde{n}_{1}\right) \\
& \cdot \exp \left[2 i k W\left(\tilde{n}_{1}^{2}-\beta^{2}\right)^{1 / 2}\right]=1 .
\end{aligned}
$$

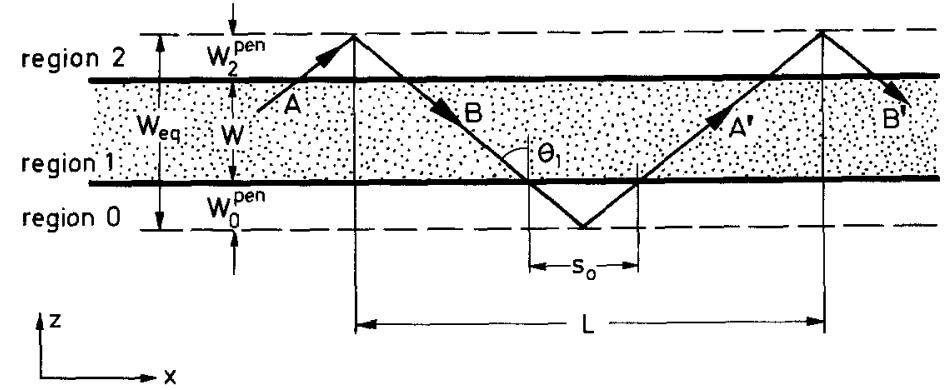

Fig. 1. Cross-section of a general slab guide. Region 1 is the slab, regions 0 and 2 are claddings. Propagation occurs by successive reflections of a plane wave at the interfaces. Due to the Goos-Hänchen effect, the width $W_{\text {eq }}$ of the zigzag path may be wider than the physical thickness $W$ of the slab 
The properties of the claddings appear here only in the $r_{1 j}$.

We recognize that this equation can be satisfied only for certain values $\beta_{m}$ of the argument $\beta$. These roots $\beta_{m}$ of $F-1=0$ are the characteristic propagation constants of the guide. The assumptions made about the cladding regions ensure that the reflection coefficients $r_{1 j}$ as functions of $\beta$ are meromorphic, i.e., their singularities are discrete poles only. As a consequence, the $\beta_{m}$ form a discrete spectrum, $m=0,1,2,3, \ldots$.

It is instructive to derive the general condition (3) also in a different way. We consider a situation where light is being coupled into the slab by some uniform excitation $A_{e}$, which may be a coupling or a scattering mechanism, or nonlinear optical generation. In solving such coupling problems one has to take into account the multiple reflections of the radiation inside the slab. Let us, therefore, specify the excitation more precisely by defining $A=t A_{e}$ as the amplitude of that plane wave, uniform along $x$ and $y$, which would be excited in the region of the slab $0<z<W$ in the absence of multiple reflections assuming, for example, that the upper slab boundary were shifted to $z \rightarrow \infty$ so that the entire space $z>0$ were filled with material $\tilde{n}_{1}$. The coefficient $t$ is then the transfer coefficient from the excitation $A_{e}$ into the medium $\tilde{n}_{1}$. Now let us return to the situation with multiple reflections: in order to find the total field in the slab, we have to sum the amplitudes of all plane waves $A$, $A^{\prime}, A^{\prime \prime}, \ldots$ going upward, and similarly of the waves $B$, $B^{\prime}, B^{\prime \prime}, \ldots$ going downward (Fig. 1). We use $A^{\prime} / A$ $=A^{\prime \prime} / A^{\prime}=\cdots=F$, with $F$ as defined in (3), and we find the total amplitude of the upward wave as

$A_{\mathrm{tot}}=t A_{e}\left(1+F+F^{2}+F^{3}+\cdots\right)=\frac{t}{1-F} A_{e}$.

From this relation, we see that freely propagating waves, i.e. waves not requiring continuous excitation $\left(A_{e}=0\right)$, can exist with nonvanishing amplitudes in the slab only if $F-1=0$. In other words, in a complex $\beta$ plane the positions $\beta_{m}$ of the freely propagating modes are the poles of the total response function $t(\beta) /[1-F(\beta)]$ of the field in the slab under a uniform external excitation $A_{e}$. These facts have been discussed thoroughly by Tamir and Oliner [8] who show how the freely propagating modes considered here are related to the continuous spectrum of the radiative modes which are also necessary for a complete representation of the fields of a general guide.

\section{Solutions of the Mode Equation}

The equation (3) permits simple solutions only in the case of a loss-less slab, $\kappa_{1}=0$, and total reflections, $\left|r_{1 j}\right|=1$. We write in this case $[6,7]$

$r_{1 j}=\exp \left(-2 i \phi_{1 j}\right)$

where the reflection phases $\phi_{1 j}\left(\beta, \tilde{n}_{1}\right)$ are known functions. We may restrict them arbitrarily to the range $-\pi / 2<\phi_{1 j} \leqq \pi / 2$. Using these phases, we obtain the solutions of (3) in implicit form as $K_{m}=0$ and

$$
\begin{aligned}
W\left(N_{m}\right)= & {\left[\phi_{10}\left(N_{m}, n_{1}\right)+\phi_{12}\left(N_{m}, n_{1}\right)\right.} \\
& +m \pi] / k\left(n_{1}^{2}-N_{m}^{2}\right)^{1 / 2}
\end{aligned}
$$

where $m=0,1,2,3, \ldots$. We can find the dispersion in the desired explicit form $N_{m}(W)$ by inverting the above equation. In practice this must be done numerically except in rare cases where the reflection phases $\phi_{1 j}$ are extremely simple functions. The phases $\phi_{i j}$ are generally different for the two possible polarizations of the light. Therefore, Eq. (6) defines two sets of modes, usually labeled as $T E_{m}$ and $T M_{m}$ modes. The integer $m$ gives directly the number of nodes in the field distribution of the $m$-th mode across the thickness $W$ of the slab [5]. The angle $\theta_{1}$ of incidence on the walls of the slab (see Fig. 1) has a characteristic value for each mode, determined by

$N_{m}=n_{1} \sin \theta_{1}$.

These facts, and in particular the field configurations, have been discussed in great detail [5] for a highindex, totally reflective slab guide, and the findings there are directly applicable to the general slab guide discussed here. For this reason, we shall focus our attention on the attenuation of the general guide. In our picture of a plane wave bouncing along the slab, we may distinguish two mechanisms contributing to attenuation: absorption in the slab material, $\kappa_{1} \neq 0$, and nontotal reflection at the slab surfaces, $\left|r_{1 j}\right|<1$. Among the reasons for nontotal reflection are: absorption in the cladding regions, refraction of light into the cladding region, and scattering of light at a slightly irregular interface.

We are interested here only in situations where the light is guided over distances substantially longer than the wavelength of light, i.e. where

$K_{m} \ll N_{m}$. 
It is clear that we can expect modes with this quality only if the guide material is not too lossy, $\kappa_{1} \ll n_{1}$, and if both reflections $r_{1 j}$ are total or nearly total. Hence we may use a perturbation method to find approximate solutions of the mode Eq. (3). We replace $F$ by a series expansion up to linear terms around the case without attenuation. Thus, we expand the transverse propagation constant in the slab

$$
\xi\left(\beta, \tilde{n}_{1}\right)=\left(n_{1}^{2}-N_{m}^{2}\right)^{1 / 2}-\delta \beta \tan \theta_{1}+\delta n_{1} / \cos \theta_{1}
$$

and we write for the reflection coefficients

$$
\begin{aligned}
r_{1 j}\left(\beta, \tilde{n}_{1}\right)= & \left(1-A_{j}\right) \exp \left[-2 i\left(\phi_{1 j}+\delta \phi_{1 j}\right)\right] \\
& +\frac{\partial r_{1 j}}{\partial \beta} \delta \beta+\frac{\partial r_{1 j}}{\partial \tilde{n}_{1}} \delta \tilde{n}_{1}
\end{aligned}
$$

We insert here $\delta \tilde{n}_{1}=i \kappa_{1}$ to account for the losses in the slab material, and $\delta \beta=\delta N_{m}+i K_{m}$ describes the attenuation $K_{m}$ in the guide and, possibly, an associated shift $\delta N_{m}$ of the modes. The two real parameters $\Delta_{j}$ (with $j=0,2$ ) in (10) are the amplitude deviations from total reflection at the $j$-th cladding region, $\Delta_{j}=1-\left|r_{1 j}\right| \ll 1$. The real quantities $\delta \phi_{1 j}$ are the deviations in the reflection phases from the case of total reflection. Thus, the first term on the right-hand side of Eq. (10) gives the reflection for the case when a homogeneous plane wave $(\delta \beta=0)$ is incident on the cladding material from a lossless $\left(\delta \tilde{n}_{1}=0\right)$ slab medium. The remaining two terms of $(10)$ are required to take into account the changes in $r_{1 j}$ occuring when the incident wave is attenuated in $x$-direction and when the wave is coming from a lossy slab medium. As we use a perturbation procedure, the derivatives in (10) and in the following equations may all be evaluated for the case of total reflections and lossless slab medium, and similarly $\theta_{1}=\arcsin \left(N_{m} / n_{1}\right)$.

A straightforward evaluation of (3), neglecting higher than linear terms in the small quantities $\Delta_{j}, \delta \phi_{1 j}$, $\kappa, K_{m}$, yields the shift of the $m$-th mode and its attenuation

$$
\begin{aligned}
& \delta N_{m}=\left(\delta \phi_{10}+\delta \phi_{12}\right) / k W_{\mathrm{eq}} \tan \theta_{1} \\
& K_{m}=\frac{W}{W_{\mathrm{eq}} \sin \theta_{1}} \kappa_{1} \\
& +\left[\Delta_{0}+\Delta_{2}-2 \kappa_{1}\left(\frac{\partial \phi_{10}}{\partial \tilde{n}_{1}}+\frac{\partial \phi_{12}}{\partial \tilde{n}_{1}}\right)\right] / 2 k W_{\mathrm{eq}} \tan \theta_{1}
\end{aligned}
$$

where

$$
W_{\mathrm{eq}}=W+\left(\frac{\partial \phi_{10}}{\partial \beta}+\frac{\partial \phi_{12}}{\partial \beta}\right) / k \tan \theta_{1} .
$$

These equations, in combination with (6), are the solutions for the general slab guide. They determine $N_{m}$ and $K_{m}$ as functions of the slab parameters $W, \tilde{n}_{1}$, and of the properties $\phi_{1 j}, \Delta_{j}$ of the claddings as seen from the slab. Before applying them to specific guides, we discuss the parameter combination $W_{\text {eq }}$ introduced in (13).

\section{The Equivalent Thickness $\boldsymbol{W}_{\mathrm{eq}}$}

In a total reflection the wave is known to penetrate slightly into the optically rarer medium. Consequently, the reflected beam is shifted some distance $s$ in the forward direction, as compared to an ideal metallic reflection. This is the Goos-Hänchen shift, indicated in Fig. 1. It has been shown [9] that for a well-collimated beam the shift associated with $r_{1 j}$ is $s_{j}=(2 / k) d \phi_{1 j} / d \beta$. Extrapolating the incident and outgoing beams from the slab region up to their point of intersection in the cladding regions, we can define the general depths of penetration $W_{j}^{\text {pen }}$ $=s_{f} / 2 \tan \theta_{1}$ into the claddings (Fig. 1). On comparing these expressions with Eq. (13), we see that the equivalent thickness is given by

$W_{\text {eq }}=W+W_{0}^{\text {pen }}+W_{2}^{\text {pen }}$.

This relationship is also shown in Fig. 1. Like the phases $\phi_{1 j}$, the equivalent thickness is generally different for the two polarizations. Moreover, $W_{\text {eq }}$ is different for the various modes $m$. For simplicity, however, we do not explicitly indicate these differences.

The Goos-Hänchen shift must also be taken into account when calculating the "hopping length" $L$ covered by the waves $A$ and $B$ in one zigzag. From Fig. 1 we find

$L=s_{0}+s_{2}+2 W \tan \theta_{1}=2 W_{\mathrm{eq}} \tan \theta_{1}$.

Because of its relation to the hopping length, the equivalent thickness $W_{\text {eq }}$ turns out to be an important quantity in many problems concerning the power flow in the guide. If we assume only a single mode to be excited, and weak attenuation $\left(\kappa_{1} \ll 1\right.$ and $\left.\Delta_{j} \ll 1\right)$ so that waves $A$ and $B$ are of equal amplitudes, we can calculate the power flow from the power density $p_{A}$ of the wave $A$. In region 1 we have 
$p_{A}=\left(n_{1} c / 8 \pi q_{1}\right)|A|^{2}$. The quantity $q_{1}$ appearing here is $q_{1}=1$ for TE polarization and $q_{1}=n_{1}^{2}$ for $T M$. The power $p_{m}$ flowing in the guide is then equal to the $x$ component of $p_{A}$, integrated over $-\infty<z$ $<+\infty$. Alternatively, we can find $P_{m}$ as the $z$ component of $p_{A}$ integrated over one hopping length $L$.

We choose the latter possibility because it does not require direct knowledge of the fields in the cladding regions. These fields enter only indirectly through $L$ and $W_{\text {eq. }}$. We find

$P_{m}=\left(c / 4 \pi q_{1}\right) N_{m} W_{\text {eq }}|A|^{2}=\left(c / 16 \pi q_{1}\right) N_{m} W_{\text {eq }}\left|\hat{V}_{1}\right|^{2}$.

Here we have introduced the peak amplitude $\left|\hat{V}_{1}\right|=|A|+|B| \approx 2|A|$ of the field in the slab. This Eq. (16) permits us to interpret the parameter $W_{\text {eq }}$ of a general slab guide as the thickness of an assumed "equivalent" guide that has walls giving ideal metallic reflections, that is filled entirely with the slab material $\tilde{n}_{1}$, and that can carry the power $P_{m}$ by a wave of the same amplitude $\left|A_{m}\right|$ propagating at the same angle $\theta_{1}$. For a guide with ideally reflecting walls, $W_{\mathrm{eq}}=W$ because $\phi_{1 j}=0$ or $\pi / 2$.

The equivalent thickness is also implicitly contained in the expression for the group velocity $v_{\mathrm{gr}}=c / N_{\mathrm{gr}}$ of the guide. The group velocity index $N_{\mathrm{gr}}$ can be derived from the dispersion $\mathrm{Eq}$. (6), neglecting the material dispersions $d n_{j} / d \lambda$, as

$N_{\mathrm{gr}}=N_{m}\left[1+W /\left(W_{\mathrm{eq}} \tan ^{2} \theta_{1}\right)\right]$

for the $m$-th mode. In the special case of $W=W_{\text {eq }}$ we obtain from this the well-known relation $v_{\mathrm{gr}} v_{\mathrm{ph}}$ $=c^{2} / n_{1}^{2}$ which is valid for waveguides with metallic walls [10].

In discussing the difference between $W_{\text {eq }}$ and $W$ for a guide consisting of a high-index film with low-index claddings of homogeneous dielectric materials $\tilde{n}_{0}$ and $\tilde{n}_{2}$, we assume now $n_{0}>n_{2}$ and weak absorptions, $\kappa_{0,2} \ll 1$. Various cases must be distinguished. In the total reflection regime (see below), $N_{m}>n_{0}$, both penetration depths $W_{j}^{\text {pen }}$ are finite and $W_{\text {eq }}>W$. If $N_{m}$ is reduced and approaches the cutoff, $N_{m} \rightarrow n_{0}$, the penetration $W_{0}^{\text {pen }}$ diverges and $W_{\text {eq }} \rightarrow \infty$. In the intermediate regime, $n_{0}>N_{m}>n_{2}$, the reflection $r_{12}$ is still total and $W_{2}^{\text {pen }}$ is finite, but $r_{10}$ is a partial reflection with $\phi_{1.0}=0$ or $\pi / 2$, so that $W_{0}^{\text {pen }}=0$. In this case, $W_{\text {eq }}$ is single-sided and $W_{\text {eq }}=W+W_{2}^{\text {pen }}$, as indicated in Fig. 2. $W_{\mathrm{eq}}$ diverges again when $N_{m} \rightarrow n_{2}$. Finally, if $N<n_{2}$, we have $\phi_{1 j}=0$ or $\pi / 2$ at both sides and $W_{\text {eq }}=W$, like in the guide with ideal metallic walls.

If the claddings are not homogeneous but layered, $W_{\text {eq }}$ may exhibit pronounced resonances whenever
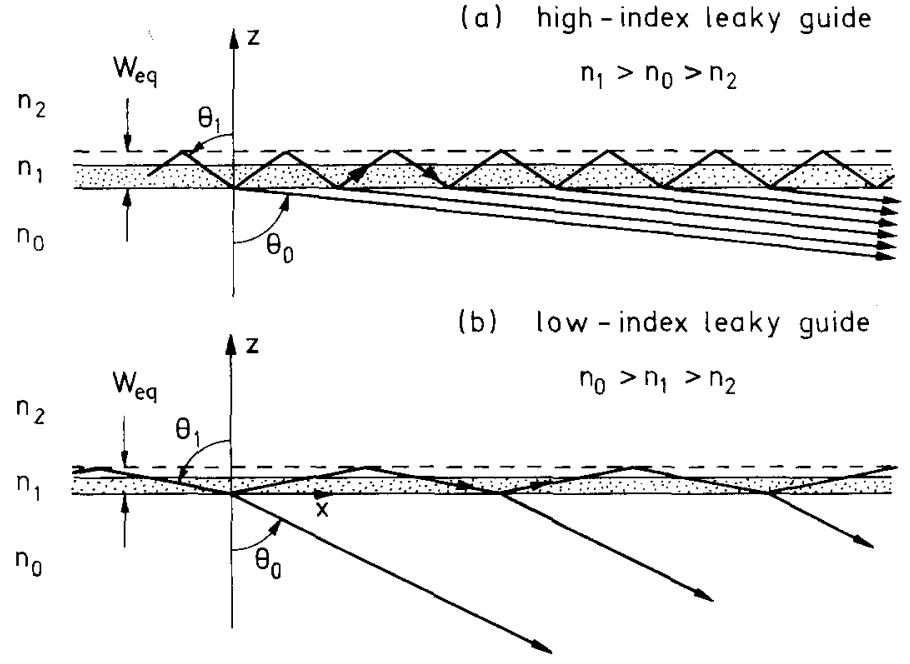

Fig. 2. Leaky guides. a) High-index slab guide with low-index claddings, operating in the Lummer-Gehrcke regime. At the interface $n_{1} / n_{0}$ light is refracted into the region $n_{0}$. The attenuation due to this leakage is lowest when the light emerges near grazing angle. b) Low-index slab guide with high-index claddings. The light in the slab travels at nearly grazing incidence, $\theta_{1} \rightarrow \pi / 2$, so that the reflection at interface $n_{1} / n_{0}$ approaches unity. The light emerges at a finite angle. In both guides, total reflection at the interface $n_{1} / n_{2}$ is assumed. Only at this surface a Goos-Hänchen effect occurs

a condition like that of Eq. (3) is satisfied for propagation in one of the layers.

It is necessary to distinguish between $W_{\text {eq }}$ and $W$ (or equivalently between the hopping lengths including or not including the shifts $s_{j}$ ) in all problems dealing with the growth $d P_{m} / d x$ of power in a mode along the guide, e.g. by absorption, scattering, or coupling. As was pointed out by Burke [11], this distinction should have been made in [5]. The results obtained there are correct, however, if $W_{\text {eq }}$ is substituted for $W$ in all expressions concerning the coupling. Another case where this necessary distinction has not been made is the theory of mode conversion in [12].

We demonstrate the role of $W_{\text {eq }}$ in calculations of $d P / d x$ by discussing the three contributions in $\mathrm{Eq}$. (12) to the attenuation $K_{m}$ of a guide: the deviations $\Delta_{0}$ and $\Delta_{2}$ from total reflection, and the absorption $\kappa_{1}$ in the slab material. The contributions from the $\Delta_{j}$ can be understood by a simple geometrical consideration (Fig. 1). The amplitude of the bouncing wave is reduced by the factor $\left(1-\Delta_{0}\right)\left(1-\Delta_{2}\right)$ in one zigzag, and this must equal the attenuation $\exp \left(-K_{m} L\right)$ over one hopping length $L$. By equating these two expressions we obtain the $\Delta_{j}$ terms of Eq. (12) in the limit $\Delta_{j} \ll 1$. The absorption $\kappa_{1}$ in the 
slab material can be expected to contribute to $K_{m}$ only in proportion to the length $2 W / \cos \theta_{1}$ of the path of the waves $A$ and $B$ through the region $\tilde{n}_{1}$ relative to the length $L$ of one zigzag. The ratio of these lengths is $W / W_{\text {eq }} \sin \theta_{1}$ and explains the first $\kappa_{1}$ term of Eq. (12). The other $\kappa_{1}$ term, containing the $\partial \phi / \partial \tilde{n}_{1}$, accounts for the interference of the waves $A$ and $B$ in the region 1 . Due to the predominantly constructive nature of the superposition of $A$ and $B$, the average electromagnetic energy density in region 1 is higher than it would be if the waves $A$ and $B$ were propagating through a bulk piece of material $\tilde{n}_{1}$, and consequently the absorption is higher. The contribution of this second $\kappa_{1}$ term varies from zero (for thick films) to a value equal to that of the first $\kappa_{1}$ term (for very thin films with symmetric claddings). In the latter limit, therefore, the attenuation of the guide is only the fraction $2 W / W_{\text {eq }}$ of the attenuation in the bulk material $\tilde{n}_{1}$, and vanishes as $W \rightarrow 0$ (see [13]).

We shall now use Eqs. (9)-(13) to discuss the guide parameters $N_{m}$ and $K_{m}$ of various slab guides that differ in their type of cladding. The main type of guide to be considered is the simple slab $\tilde{n}_{1}$ sandwiched between two homogeneous cladding materials $\tilde{n}_{0}=n_{0}+i \kappa_{0}$ and $n_{2}=\tilde{n}_{2}+i \kappa_{2}$. For this arrangement, and ideally smooth interfaces, we have the reflection coefficients $[6,7]$

$r_{1 j}=\left(\Gamma_{1}-\Gamma_{j}\right) /\left(\Gamma_{1}+\Gamma_{j}\right)$

where $\Gamma_{j}=\left(n_{j}^{2}-\beta_{m}^{2}\right)^{1 / 2} / q_{j}^{2}$, and where $q_{j}=1$ for $T E$ polarization, $q_{j}=n_{j}^{2}$ for $T M$ polarization, $j=0,1,2$.

\section{High-Index, Totally Reflecting Guide}

If all three regions, $0,1,2$, are lossless and if both $n_{0}$ and $n_{2}$ are smaller than $n_{1}$, total reflections will occur at the surfaces of the slab, provided $\theta_{1}$ exceeds the critical angles $\theta_{c, j}=\arcsin \left(n_{j} / n_{1}\right)$ at both surfaces. This is guaranteed if $N_{m}>n_{0}$ and $N_{m}>n_{2}$. Then both $r_{1 j}$ lie on the unit circle in the complex $r$ plane so that $\Delta_{j}=0$, and the attenuation vanishes, $K_{m} \rightarrow 0$. For this reason, the high-index guide operating at $N_{m}>n_{0}, n_{2}$ is the slab guide of greatest practical importance. It has been extensively covered in the literature $[1,2,5,7,10,13]$ and need not be treated in detail here. An example of the dispersion of this guide is seen in the right half of Fig. 3, where we have plotted $W\left(N_{m}\right)$ for the first few $T E_{m}$ modes of an assumed slab guide of index $n_{1}=1.515$, deposited on a substrate of $n_{0}=1.50$, and with free space $n_{2}=1$ above it.

We may use our formula Eq. (12) to find for this guide the attenuation which will result from absorption in any one of the three materials. Let us consider the case of a weakly absorbing cladding material $\tilde{n}_{0}=n_{0}+i \kappa_{0}$ with $\kappa_{0} \ll n_{0}$. This problem is of interest for the application of the optical waveguide in ATR spectroscopy, and was treated in a different way by Midwinter [14]. We assume TE polarization. From Eq. (18) we find with $\delta \tilde{n}_{0}=i \kappa_{0}$ the changes of $r_{10}$

$$
\begin{aligned}
\Delta_{0} & =-\operatorname{Re}\left(\frac{1}{r_{10}} \frac{\partial r_{10}}{\partial \tilde{n}_{0}} \delta \tilde{n}_{0}\right)=\frac{2 \kappa_{0} n_{0}}{n_{1}^{2}-n_{0}^{2}} \frac{\left(n_{1}^{2}-N_{m}^{2}\right)^{1 / 2}}{N_{m}^{2}-n_{0}^{2}} \\
\delta \phi_{10} & =-\frac{1}{2} \operatorname{Im}\left(\frac{1}{r_{10}} \frac{\partial r_{10}}{\partial \tilde{n}_{0}} \delta n_{0}\right)=0 .
\end{aligned}
$$

Here $\mathrm{Re}$ and $\mathrm{Im}$ are real and imaginary parts, respectively. Thus, the mode is not shifted in this case. The attenuation of the guide, relative to the attenuation of a freely propagating wave in medium $\tilde{n}_{0}$, is

$$
\frac{K_{m}}{\kappa_{0}}=\frac{n_{1}^{2}-N_{m}^{2}}{n_{1}^{2}-n_{0}^{2}} \frac{n_{0}}{N_{m}} \frac{1}{k W_{\mathrm{eq}}\left(N_{m}^{2}-n_{0}^{2}\right)^{1 / 2}} .
$$

In discussing this expression, it should be noted that $N_{m}$ and $W_{\text {eq }}$ are not independent. In the limiting case of a thick guide operating in a low-order mode so that $N \rightarrow n_{1}$, we find

$$
\frac{K_{m}}{\kappa_{0}}=\frac{\pi^{2} n_{0}}{n_{1}\left(n_{1}^{2}-n_{0}^{2}\right)^{3 / 2}} \frac{(m+1)^{2}}{\left(k W_{\mathrm{eq}}\right)^{3}} .
$$

From this $W_{\mathrm{eq}}^{-3}$ dependence we can conclude that for ATR spectroscopic applications a low-order mode in a thick film will yield poor sensitivity. To put it in another way, the influence of the cladding losses on the total attenuation in a guide can be made negligibly small by moderately increasing the slab thickness. As an example, we consider a guide of $n_{0}=1.50 ; n_{1}=1.65 ; n_{2}=1.0 ; m=0$, guiding light of $\lambda=0.633 \mu \mathrm{m}$. For a film thickness of $W=3 \mu \mathrm{m}$, the equivalent thickness is $W_{\text {eq }} \approx 3.15 \mu \mathrm{m}$ and the ratio Eq. (20) of guide attenuation to bulk attenuation in the cladding material is less than $10^{-3}$ for the fundamental $T E_{0}$ mode.

The other extreme is that of a very thin film, operating near cutoff. Assuming $n_{0} \geqq n_{2}$, we have $N_{m} \rightarrow n_{0}$ in this case and the ratio Eq. (19) approaches unity if $n_{0}>n_{2}$, or 0.5 if $n_{0}=n_{2}$. Both situations yield high sensitivity for the ATR application as a consequence 
of the deep penetration of the field into the cladding regions near cutoff. With asymmetric claddings, $n_{0}>n_{2}$, the field penetrates deeply only into $n_{0}$, but in the symmetric case it penetrates into both $n_{0}$ and $n_{2}$.

\section{High-Index Leaky Guide (Lummer-Gehrcke Modes)}

It is characteristic of the high-index guide treated in the previous section that each mode (except $m=0$ in a symmetric guide) has a finite cutoff thickness $W_{c}$. At this thickness, the angle $\theta_{1}$ of incidence of the plane waves $A$ and $B$ approaches the critical angle $\theta_{c}$ of total internal reflection at one or both of the interfaces. If the thickness of the guide is below the cutoff for a given mode, the guide is said to be unable to support that mode. However, this is only partially true. The general mode Eq. (3) has solutions also when $W<W_{c}$, corresponding to $\theta_{1}<\theta_{c}$ or equivalently to $N_{m}<n_{0}$, assuming $n_{0} \geqq n_{2}$. The reflection $r_{10}$ (and possibly also $r_{12}$ ) is no longer total. In each of the reflections $r_{10}$ a fraction of the incident light is refracted out of the slab into the substrate $n_{0}$, as indicated in Fig. 2a. The refracted light propagates away from the guide at the refraction angle $\theta_{0}=\arcsin \left(N_{m} / n_{0}\right)$ and constitutes a radiative loss to the guided wave. The loss can be so large that the mode ceases to exist for practical purposes.

A guide having this type of radiative loss is called a leaky guide [8]. The best-known example for a highindex slab guide operated in this leaky regime, $N<n_{0}$, is the Lummer-Gehrcke interferometer plate, known in high-resolution spectroscopy [15]. Therefore, the modes in a high-index guide below cutoff are called "Lummer-Gehrcke" (L.G.) modes. These modes are readily observed as the various "orders" in the usual application of the L.G. interferometer [15].

We calculate the dispersion of the L.G. modes from Eq. (6). We assume an asymmetric guide, $n_{0}>n_{2}$, consisting of all lossless materials. The reflection phases are $\phi_{10}=0 ; \phi_{12}=\arctan \left(\Gamma_{2} / i \Gamma_{1}\right)$, and we find

$W\left(N_{m}\right)=\left[m \pi+\phi_{12}\left(N_{m}\right)\right] / k\left(n_{1}^{2}-N_{m}^{2}\right)^{1 / 2}$.

These functions are illustrated in the left half of Fig. 3 for the same guide that was considered earlier at $N>n_{0}$. We see that the dispersion curves of the L.G. modes join continuously the ordinary, total-reflection modes of the same guide, although there is a discontinuity in the slope of the $W(N)$ curves at the cutoff. The attenuation $K_{m}$ of the L.G. modes follows

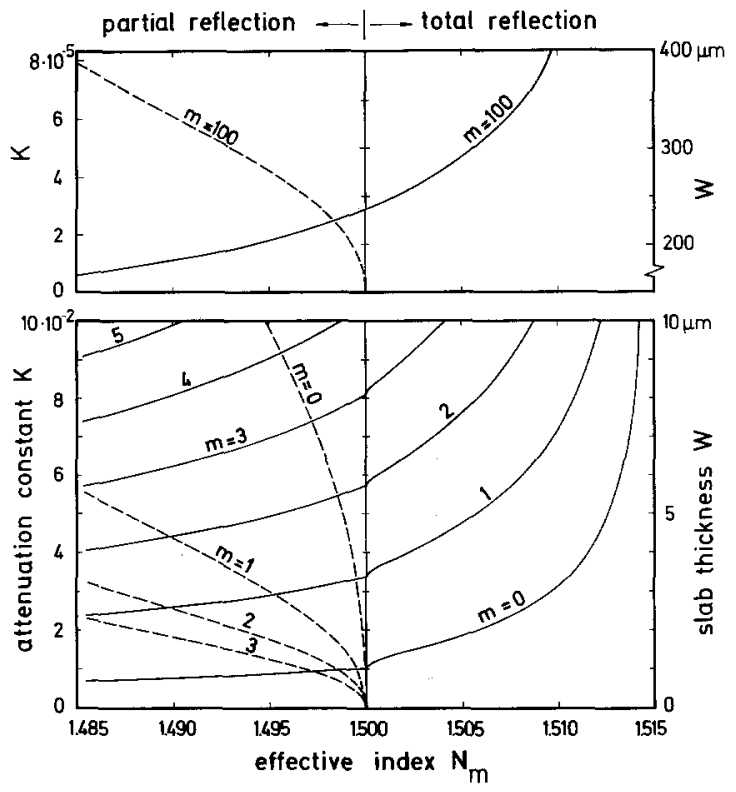

Fig. 3. Dispersion and attenuation of TE modes in a high-index slab guide of composition $n_{0}=1.500, n_{1}=1.515$, and $n_{2}=1.000$ The parameter at the curves is the mode number $m$. - - slab thickness $W$ vs. effective index $N_{m}$; ----- attenuation constant $K_{m}$ vs. $N_{m}$. For $N_{m}>n_{0}$ (right half of figure) reflection is total at both surfaces of the slab, and the attenuation vanishes. For $N_{m}<n_{0}$ (left half of figure) the reflection at the interface $n_{1} / n_{0}$ is partial, and the guide is leaky (Lummer-Gehrcke regime of operation)

from Eq. (12). The deviation parameter $\Delta_{0} \ll 1$ is obtained by a series expansion of $r_{10}$ in the point $\beta=n_{0}$ as $\Delta_{0} \approx 2 \Gamma_{0} / \Gamma_{1}$, and

$K_{m}=\frac{q_{1}}{q_{0}} \frac{\left(n_{0}^{2}-N_{m}^{2}\right)^{1 / 2}}{N k W_{\mathrm{eq}}}$.

Here $W_{\mathrm{eq}}$ is the one-sided equivalent thickness. The attenuation $K_{m}$ has also been plotted in Fig. 3 as a function of $N_{m}$. The values of $K_{m}$ are small $\left(K_{m}<0.02\right)$ for the entire range plotted, so that is it justified to speak of all these modes as guided modes. Yet, with thin films, the absolute attenuation values of these modes are too high to be of practical interest for light guiding. For example, a value of $K=0.01$ corresponds to an attenuation of $10^{4} \mathrm{~dB} / \mathrm{cm}$ in the visible. If the film thickness is increased, giving correspondingly higher modes at a given $N$, the attenuation decreases as $W^{-1}$. For a film thickness near $W=238 \mu \mathrm{m}$, for example, the attenuation of the $m=100$ mode varies between 0 and not more than $6 \mathrm{~dB} / \mathrm{cm}$ in the narrow $N$ range $n_{1}-N_{100}<1.9 \times 10^{-4}$ where the $m=100$ mode is the highest existing one (Fig. 3). 
The above theory might appear suitable for a quantitative description of the tapered film coupler of Tien and Martin [16], which is a high-index leaky guide in the region beyond the cutoff point, $x>x_{c}$. A closer study of the fields in the vicinity of the cutoff point $x_{c}$ shows, however, that the equivalent thickness $W_{\mathrm{eq}}$ diverges like $\left(x_{c}-x\right)^{-1}$ in the region $x<x_{c}$. The field there penetrates deeply into the substrate so that at $x_{c}$, the feeding point of the leaky guide, only a small fraction of the total power is left in the film itself. Because the present theory can treat only that fraction, it cannot give an adequate description of the tapered film coupler.

\section{Low-Index Leaky Guide}

In a slab whose refractive index $n_{1}$ is lower than the cladding index $n_{0}$ there is only partial reflection of the zigzag bouncing wave for any angle $\theta_{1}$ of incidence, and light leaks into $n_{0}$. For generality, we assume an asymmetric guide in which such a condition of partial reflection exists at the interface $n_{1} / n_{0}$, but there is total reflection assumed at the other interface $n_{2} / n_{1}$ (see Fig. 2b). All materials should be lossless. This guide is characterized by

$n_{2}<N<n_{1}<n_{0}$.

The results obtained for this asymmetric guide can later be extended directly to a symmetric guide [27] $\left(N<n_{1}<n_{0}=n_{2}\right)$ in which light leaks into both claddings.

Light can be guided with low attenuation in the lowindex slab if the angle $\theta_{1}$ is close to grazing incidence, because the reflection coefficient then approaches unity. Mathematically, this occurs when $\Gamma_{1} \rightarrow 0$. In this limit, we find from Eq. (18) $\Delta_{0}=2 \Gamma_{1} / \Gamma_{0}$; $\phi_{10}=\pi / 2 ; \quad \phi_{12} \approx \pi / 2-i \Gamma_{1} / \Gamma_{2}$, and the dispersion and attenuation follow from Eqs. (6) and (12),

$$
\begin{aligned}
& N_{m}=n_{1}-\frac{\pi^{2}}{2 n_{1}} \frac{(m+1)^{2}}{\left(k W_{\mathrm{eq}}\right)^{2}}, \\
& K_{m}=\frac{q_{0} \pi^{2}}{q_{1} n_{1}\left(n_{0}^{2}-n_{1}^{2}\right)^{1 / 2}} \frac{(m+1)^{2}}{\left(k W_{\mathrm{eq}}\right)^{3}}
\end{aligned}
$$

where

$$
k W_{\text {eq }}=k W+\left(q_{2} / q_{1}\right)\left(n_{1}^{2}-n_{2}^{2}\right)^{-1 / 2}
$$

is the single-sided equivalent thickness in the limit $N_{m} \rightarrow n_{1}$. The relations Eqs. (23) and (24) are illustrated in Fig. 4 for a specific numerical example. These

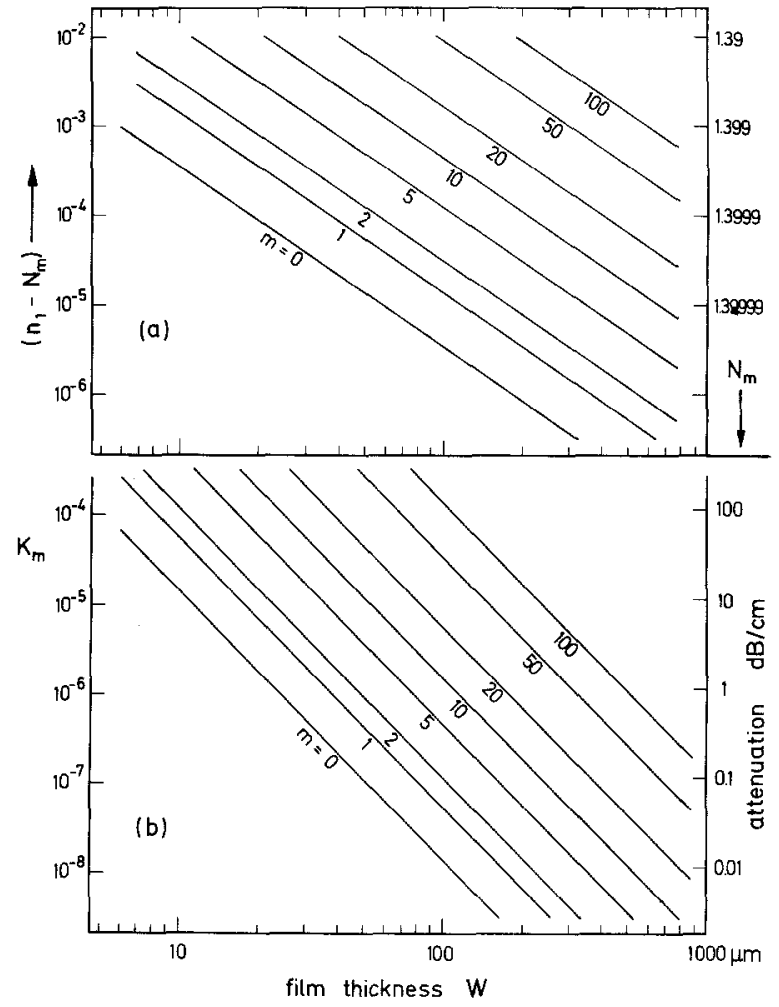

Fig. 4. a) Dispersion and b) attenuation of a low-index leaky guide plotted as functions of the film thickness $W$. The parameter at the curves is the mode number $m$. Only TE modes are shown. The refractive indices are $n_{0}=1.50, n_{1}=1.40$, and $n_{2}=1.00$. The wavelength is $\lambda=0.633 \mu \mathrm{m}$

curves are representative, however, of a fairly wide range of indices found in guides of practical interest. As in the high-index guide, the effective index $N_{m}(W)$ increases monotonically with the film thickness and approaches asymtotically the index of the slab material. This is a consequence of the nearly grazing incidence, $\theta_{1} \rightarrow \pi / 2$. It is remarkable that $N_{m}$ is entirely independent of $n_{0}$, and almost independent of $n_{2}$ which enters Eq. (23) only through $W_{\mathrm{eq}}$. The attenuation $K_{m}$ decreases fast, approximately as $W^{-3}$, with increasing film thickness (Fig. 4b). For a moderately thick film of $W=25 \mu \mathrm{m}$, for example, the attenuation by leakage is only $0.8 \mathrm{~dB} / \mathrm{cm}$ in the fundamental mode of the guide shown in Fig. 4. This value is comparable to the loss reported for many thin-film optical guides of the high-index, totally reflective type. Thus, a low-index film with high-index claddings is potentially useful for such practical light-guiding applications as a waveguide laser [17] in planar form, provided the film thickness 
is $W \gtrsim 40 \lambda$. Such a guide would properly be called a thick-film guide. For possible applications it may be of interest to note that the higher order modes are considerably more attenuated than the $m=0$ fundamental mode, $K_{m}$ being proportional to $(m+1)^{2}$. The losses of the TM modes are slightly higher (by a factor of $n_{0}^{2} / n_{1}^{2}$ ) than those of the corresponding $T E$ modes. Furthermore, the attenuation by leakage depends on the difference in index between the film and the cladding on the leaky side. A larger difference gives a higher reflectivity and hence lower attenuation.

It is instructive to compare the attenuation Eq. (24) of the leaky guide with the expression Eq. (20) for the attenuation caused by a lossy cladding on a highindex, totally reflective guide. Except for constant factors, we have in both equations the proportionality

$K_{m} \sim(m+1)^{2} /\left(k W_{\mathrm{eq}}\right)^{3}$,

valid in the limit of grazing incidence, $\theta_{1} \rightarrow \pi / 2$. In both cases, power is continuously being lost from the slab $\tilde{n}_{1}$ into the substrate $\tilde{n}_{0}$. In one case it is absorbed there, while in the other it is propagating away. The amount of power entering the substrate is proportional to the square $\left|V_{10}\right|^{2}$ of the field amplitude at the interface $n_{1} / n_{0}$. This amplitude is $V_{10}=t_{10} A$, where $t_{10}=1+r_{10}$ is the amplitude transmission coefficient of the interface $n_{1} / n_{0}$. It can be shown that near grazing incidence, $N \rightarrow n_{1}$,

$\left|t_{10}\right|^{2} \approx \Delta_{0}^{2} \approx \frac{\left(2 \pi q_{1} / q_{0}\right)^{2}}{\left|n_{0}^{2}-n_{1}^{2}\right|}\left(\frac{m+1}{k W_{\mathrm{eq}}}\right)^{2}$.

Further, we notice from Eq. (16) that $|A|^{2} \sim P /\left(k W_{\text {eq }}\right)$. Thus we recognize that both $\left|V_{10}\right|^{2}$ and $K_{m}$ must exhibit the proportionality Eq. (26). This dependence must also be expected to hold for scattering losses from roughness [2] of the interface $n_{1} / n_{0}$ in guides of either type, because for a given roughness the scattered power is also proportional to $\left|V_{10}\right|^{2}$. Furthermore, Eq. (26) must hold for the TE modes in a dielectric slab $n_{1}$ with claddings of a real metal $\tilde{n}_{0}$ in a wide range of optical constants $\tilde{n}_{0}$, provided $\left|n_{0}+i \kappa_{0}\right| \gg 1$. This expectation is confirmed by the detailed calculations of Takano and Hamasaki [18]. It is a trivial consequence of Eq. (26) that light can be propagated with negligible attenuation along any transparent dielectric slab, regardless of the type of cladding, provided only that the slab is thick enough. What is not trivial about Eq. (26) is that it shows how the attenuation $K_{m}$ varies in this transition regime between strictly guided and quasi-free optical propagation.

So far, we have discussed a slab guide that is leaky on only one side. If $n_{1}<n_{2}$ in addition to $n_{1}<n_{0}$, leakage will generally occur on both sides. In this case, we have $\phi_{10}=\phi_{12}=\pi / 2$ and $W=W_{\text {eq }}$. The dispersion Eq. (23), giving $N_{m}(W)$, remains unchanged. In Eq. (24), describing the attenuation $K_{m}$, we must add a second leakage term containing $n_{2}$ instead of $n_{0}$ in order to account also for the leakage into $n_{2}$. In the case of a symmetric cladding, $K_{m}$ simply doubles.

The low-index, leaky guide discussed here is the twodimensional equivalent of the hollow-fiber optical guide investigated by Marcatili and Schmeltzer [19]. Despite their different geometries and the resulting differences in the field configurations, these two types of guide have in common the $W^{-3}$ dependence of their attenuations, if we identify $W$ with the radius of the fiber. For this low-index optical fiber it is known that minor deviations from straightness cause a drastic increase in attenuation. We must expect the same to be true of our planar guide.

\section{Experiments on Low-Index Leaky Guides}

As with high-index guides, the modes of a low-index leaky guide can be visually observed through their so-called " $m$-lines" $[20]$. These lines result from the in-plane scattering of light out of one excited mode into other directions of the same mode and of other modes. We have observed the $m$-lines of low-index guides formed simply by placing a flat piece of glass at a distance of a few microns parallel to the base of a symmetric coupling prism, and shining a $\mathrm{He}-\mathrm{Ne}$ laser beam on the prism base at an angle slightly below the critical angle. The low-index guiding film is here represented by the air gap between the base of the prism and the glass plate. A quantitative study on the field configurations of these modes has recently been made by Zeidler [21].

We performed an experiment to measure the attenuation of the leaky guide as a function of the film thickness $W$. The guide consisted of a low-index liquid film (n-butanol, index $n_{1}=1.398$ ) held by capillary action between two plates of fused quartz $\left(n_{0}=n_{2}=1.458\right)$. The use of a liquid film allowed us to vary the thickness $W$ continuously by means of a micrometer screw (Fig. 5). Both ends of the guide were terminated by microscope cover slides to pro- 


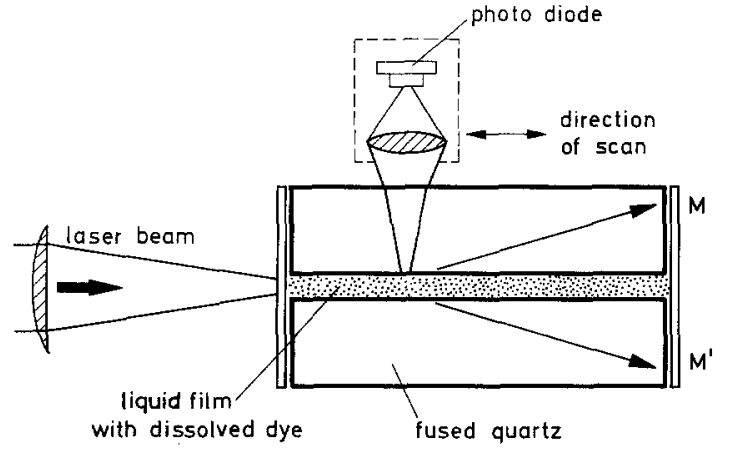

Fig. 5. Experimental set-up for measuring the attenuation of a leaky guide. The guide consists of a liquid film (n-butanol, refractive index $n_{1}=1.397$ ), cladded by two plates of fused quartz (index $\left.n_{0}=1.458\right)$. The guided He-Ne laser beam $\left(T M_{0}\right.$ mode) is made visible from the side by the fluorescence of a dissolved dye

vide optically flat windows. The beam of a $\mathrm{He}-\mathrm{Ne}$ laser was focused with a cylindrical lens on the entrance window and coupled into the guide. By adjusting the spot size, the position, and the direction of the incident beam we could selectively excite the various modes, as described by Shubert and Harris [1] for a high-index guide. The path of the light in the guide was made visible by the fluorescence of a dye (3,3'diethyl-2,2' thiadicarbocyanine iodide) dissolved in the liquid.

This set-up enabled us to observe the trace of the light guided in the fundamental mode along the entire length of the guide $(75 \mathrm{~mm})$ at large film thicknesses $(W>50 \mu \mathrm{m})$. When the thickness of the film was reduced, however, the intensity of the fluorescent light showed a rapid decrease toward the end of the guide. Simultaneously, the leaky waves appeared at $M, M^{\prime}$. We determined the attenuation of the guide by using a detector to pick up some of the fluorescent light from the side through one of the quartz plates, and then scanning the detector along the guide. The logarithm of the detected signal was plotted against the position $x$ on the guide, see Fig. 6 . The slope of these curves is a direct measure of the attenuation $\alpha$, and Fig. 7 shows the measured dependence of $\alpha$ on the film thickness. For large thicknesses $\alpha$ approaches a constant value which represents the absorption of the dye solution. In order to obtain the attenuation due to leakage alone, all measured values were corrected by this constant. Within the accuracy of the experiment, the resulting data agree well with the $W^{-3}$ line expected theoretically from Eq. (24). Because leakage occurs on both sides this line gives twice the value of Eq. (24). The largest source of error

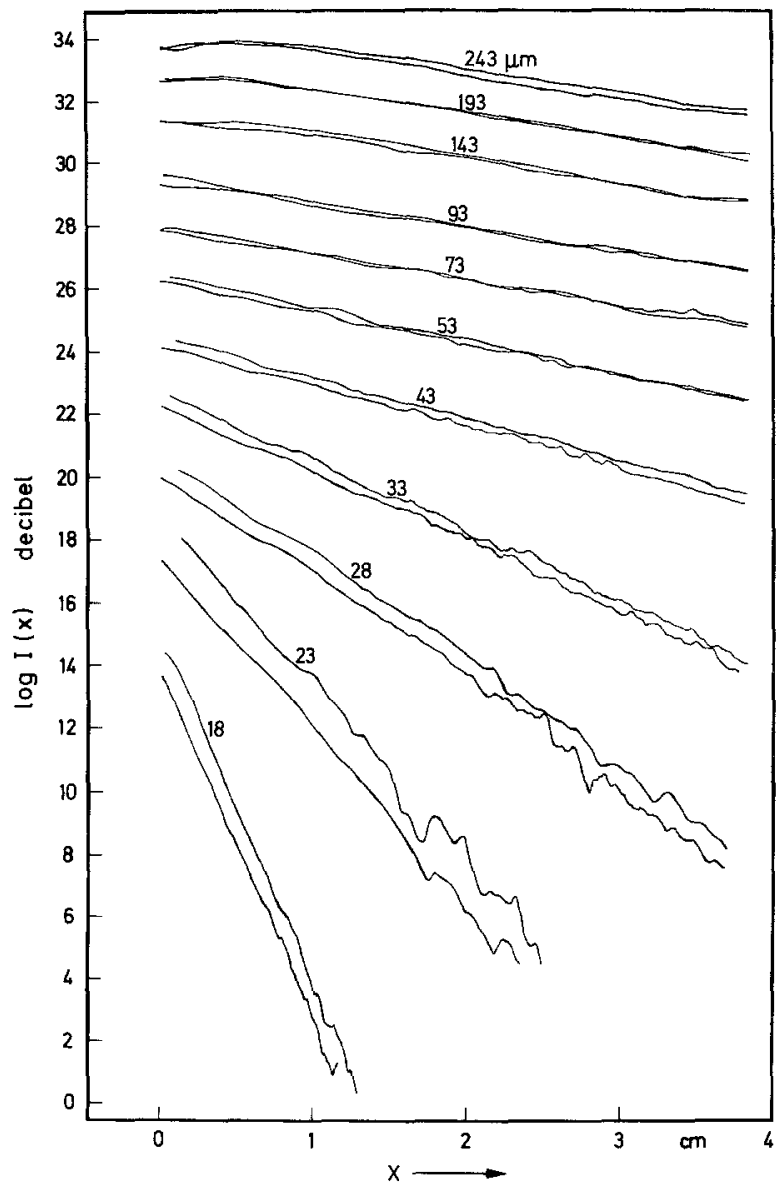

Fig. 6. Measured intensity $I(x)$ of the light beam guided in the leaky guide shown in Fig. 5 versus position $x$ on the guide. The parameters identifying the curves are the film thickness $W$ in $\mu \mathrm{m}$. For each thickness $W$ two curves are plotted, corresponding to scanning in positive and negative $x$ direction. The slope of a curve gives directly the attenuation

in these measurements was the determination of the absolute film thickness $W$. This was done by observing the angular positions of the interference fringes of a Fabry-Perot, formed by reflective coatings on small areas of the inner surfaces of the quartz plates, and also by using spacers of known thickness.

As stated in the previous section, the attenuation in a thick-film guide is fairly insensitive to the optical constants of the cladding materials. In order to demonstrate this experimentally, we replaced one of the quartz plates by a glass plate (Schott neutral density filter glass NG3) having a bulk absorption of $1000 \mathrm{~dB} / \mathrm{cm}$. Yet, at a fixed spacing of $W=29 \mu \mathrm{m}$ we measured an attenuation (corrected for the absorption of the dye) of only $1.5 \mathrm{~dB} / \mathrm{cm}$, compared to 


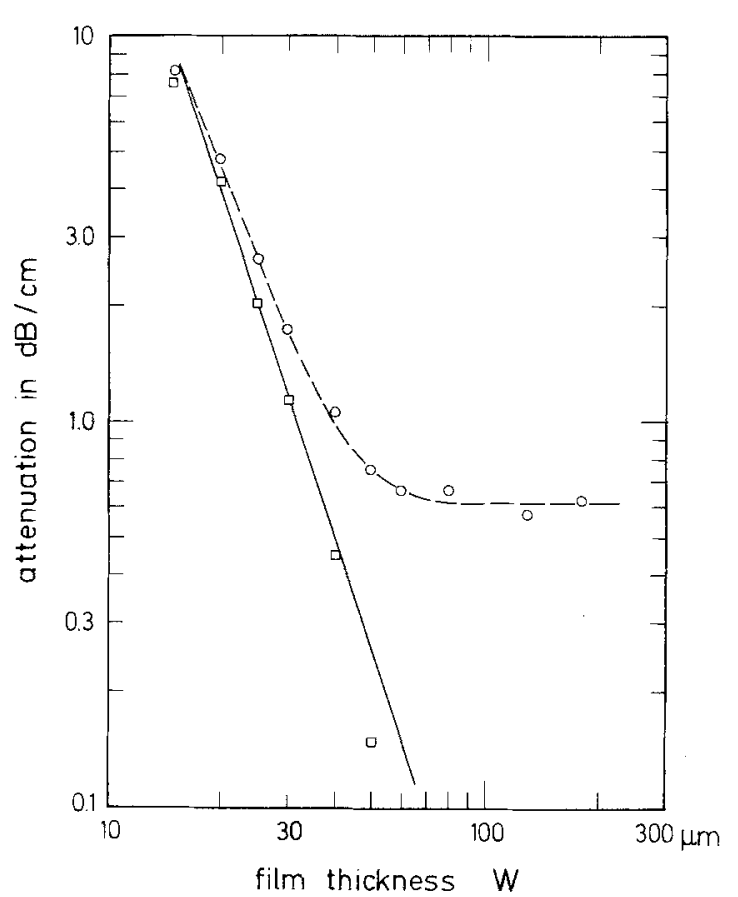

Fig. 7. Measured attenuation of the leaky light-guide of Fig. 5 as a function of the film thickness $W$. The points $\bigcirc$ are the values actually measured. The points $\square$ have been corrected by the constant attenuation measured at large $W$, which is due to the absorption of the dissolved dye. The straight line is the theoretical curve, calculated from Eq. (24). There is no adaptable parameter in the theory

$1.23 \mathrm{~dB} / \mathrm{cm}$ with nonabsorbing quartz on both sides. In a similar experiment, we replaced one of the highly polished quartz plates by a quartz plate with a finely ground surface, opaque to the eye due to scattering. With this surface as one of the waveguide walls, a (corrected) attenuation of $1.27 \mathrm{~dB} / \mathrm{cm}$ was determined at $W=29 \mu \mathrm{m}$. These results show that the cladding has indeed little influence on the attenuation of the fundamental mode in this thick-film guide.

\section{Leaky Wave Coupling}

When the leak rate is small, $K_{m} \ll 1$, the light leaking out of the film into the high-index cladding $n_{0}$ has the form of a beam well collimated in the $x z$ plane. This leakage beam, hitherto considered as a loss to the guiding, can be utilized to couple light out of the guide or, by inversion, to couple a light beam into the guiding film [28]. Fig. 8 shows schematically a coupler based on this effect. Because couplers like this are reversible [22], it is sufficient to explain its

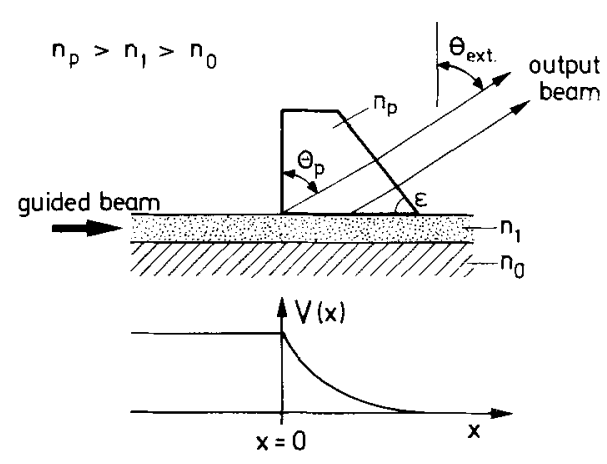

Fig. 8. A leaky wave coupler shown for output from a guide. The local amplitude $V(x)$ of the wave remaining on the film decays exponentially as $\exp \left(-x / l_{m}\right)$. By suitable choice of the prism angle $\varepsilon$ the external coupling angle $\theta_{\text {ext }}$ can be made independent of wavelength in first-order approximation

operation for output from the guide. The guiding film $n_{1}$ has a higher index than the substrate $n_{0}$, so that the guide is of the totally reflective type in the region $x<0$ where the top cladding is free space. In the region $x>0$ the film is in optical contact with a material (prism) whose index $n_{p}$ is higher than the index $n_{1}$ of the film. Therefore, the guide is leaky in $x>0$, and an output beam emerges from the guide into the prism and thence into free space. The amplitude $|V(x)|$ of the wave remaining in the guide decreases exponentially, as indicated in Fig. 8. The angle between the output beam and the normal of the film is (Fig. 8)

$\theta_{p}=\arcsin \left(N_{m} / n_{p}\right)$.

This leaky wave coupler may be regarded as a prismfilm coupler $[5,7,22]$ which has been simplified by omitting the coupling gap. Gapless couplers were used in some of the early work on planar light guides [23], but the coupling efficiencies achieved were low. If the leaky wave coupler is seen as a limiting case of the prism-film coupler, we must expect the gapless coupler to give the same high coupling efficiency as the coupler with a gap, i.e. $\eta \approx 0.80$. The results obtained above for the leakage from the guide allow us to formulate a condition for realizing this high efficiency.

The "coupling length" $l_{m}$ is used as the principal parameter characterizing a uniform coupler [7]. The coupling length is that distance along the guide within which the amplitude of the $m$-th guided mode decays by leakage to $1 / e$. From Eq. (24) we have

$l_{m}=\frac{q_{1} n_{1}\left(n_{p}^{2}-n_{1}^{2}\right)^{1 / 2}}{q_{p} k \pi^{2}} \frac{\left(k W_{\mathrm{eq}}\right)^{3}}{(m+1)^{2}}$ 
where $W_{\mathrm{eq}}$ is the one-sided equivalent thickness calculated with $n_{p}$ as the cladding index. The approximations made in the derivation of Eq. (24) also limit the validity of Eq. (29) to cases where $k l_{m}>1$. This is satisfied for the lower modes in not-too-thin films.

When using a nonabsorbing leaky guide as an output coupler, we can always achieve an output efficiency close to unity, $\eta \rightarrow 1$, by making the coupler long enough so that all guided power leaks out. Along the initial coupling length $0<x<l_{m}$, for example, a fraction of $\left(1-e^{-2}\right) \approx 0.86$ of the power is coupled out. For input coupling, the efficiency depends on the relative dimensions of beam radius and coupling length. It has a broad maximum of $\eta=0.801$ near $l_{m}=1.48 w$, where $w=w_{0} / \cos \theta_{p}$ is the waist radius of the gaussian input beam, measured along the guide [7]. For both input and output, therefore, the problem of designing an optimum leaky coupler is one of achieving the required value of $l_{m}$. A numerical example will show that this is possible with reaonable values of all parameters: we consider the $T E_{0}$ mode of $\lambda=0.63 \mu \mathrm{m}$ in a film of $n_{1}=1.5095$ (Schott K 7 glass) on a substrate of $n_{0}=1.4986$ (Schott K 11), and a coupling prism of flint glass $n_{p}=1.6185$ (Schott SK 16). With this combination of materials, we have $n_{1}-N_{0}=2.6 \times 10^{-3}$, and a coupling angle of $\theta_{p}=69^{\circ}$. In order to accept a typical input beam of waist radius $w_{0}=100 \mu \mathrm{m}$ (corresponding to a full angular aperture of $4 \times 10^{-3}$ in air), we require the optimum coupling length $l_{0}=0.41 \mathrm{~mm}$ for the $T E_{0}$ mode. According to Eq. (29) this can be achieved with a film thickness of $W \approx 3 \mu \mathrm{m}$. This value is reasonable for practical applications because the guide can support in addition to the $T E_{0}$ mode only the $T E_{1}$ and the two lowest $T M$ modes.

The optimum film thickness does not differ much for other values of the refractive indices. Thus, for any application where a film thickness of about $5 \lambda$ can be used, the gapless coupler appears to offer the simplest solution to the coupling problem. We have made a number of such couplers by cementing tiny polished blocks $\left(1 \times 2 \times 2 \mathrm{~mm}^{3}\right)$ of flint glass onto a $3 \mu \mathrm{m}$ guide with a cement [24] matching the prism index.

In the prism-film coupler, the theoretical maximum coupling efficiency of $\eta=0.801$, valid for a uniform gap width, can be improved to almost $\eta=1$ by tapering the coupling gap [22]. For the leaky wave coupler, a similar optimization must be possible. It would involve the use a film of nonuniform thickness
$W=W(x)$, designed to make the leak rate a function of position $x$, conforming to the power distribution in the beam.

Changing our point of view, we may regard the ordinary prism-film coupler as a generalized leaky wave coupler in which the leakage is reduced by the introduction of a low-index gap. The index and width of the gap can now be seen as a set of two parameters by means of which the leakage can be controlled to give a specified, long coupling length independent of the properties of the film. By computing the deviations $\Delta_{0}$ and $\delta \phi_{10}$ for the reflection at the prism-gap combination, the theory of the prismfilm coupler can be worked out quantitatively from our general Eqs. (11) and (12) for the dispersion and attenuation of a guide.

To conclude this section, we note that the gapless leaky wave coupler has intrinsically a broad optical bandwidth. This is because the effective index $N_{m}$ deviates very little from the film index $n_{1}$ for typical coupling situations $\left(l_{m}>100 \mu \mathrm{m}\right)$. Therefore, the internal coupling angle $\theta_{p}$, given by Eq. (28), also varies very little with wavelength. In fact, the dependence of $\theta_{p}$ on wavelength is mainly due to the difference in material dispersions $d n / d \lambda$ between prism and film, with only a minor contribution from the finite width of the guide. For practical broad-band operations, though, the external coupling angle $\theta_{\mathrm{cxt}}$ (Fig. 8) must be independent of wavelength. In favorable cases, this can be achieved by a proper choice of the prism angle $\varepsilon$. An analysis of the problem shows that $d \theta_{\mathrm{ext}} / d \lambda=0$ if

$\varepsilon=\theta_{p}-\arctan$

$\left\{\left[1-D_{1} / D_{p}+2\left(n_{1}-N_{m}\right) /\left(\lambda N_{m} D_{p}\right)\right] \tan \theta_{p}\right\}$.

Here $D_{j}=n_{j}^{-1} d n_{j} / d \lambda$ is the logarithmic dispersion of material $n_{j}$, and all quantities in the above formula are computed at the center wavelength of the band to be coupled. Let us calculate the angle $\varepsilon$ of the broad-band prism in the example considered earlier. The film and prism materials there (K 7 and SK 16) had already been selected so that their dispersion values $\left(D_{1}=-0.0249 \mu^{-1}\right.$ and $\left.D_{p}=-0.028 \mu^{-1}\right)$ nearly cancel in Eq. (30), giving an optimum prism angle of $\varepsilon=80^{\circ}$. No exact determination of the band width of this coupler has been made; the band width is probably limited by the fast variation of $K_{m}$ with $k^{-3}$. We estimate that a range of wavelengths with $\lambda_{\min }: \lambda_{\max }$ approximately $1: 3$ can be coupled, tolerating a $3 d B$ reduction of the efficiency at the extreme wavelengths. 
Finally, we compare this broad-band coupler with the one proposed by Midwinter [25]. In the design of both couplers one has to compensate the dispersion of the materials with each other and with a geometrical dispersion resulting from the fact that the film thickness, measured in units of the wavelength, is not a constant. This latter dispersion can be considerably larger than the material dispersions [4].

Midwinter reduced the influence of the geometrical dispersion by choosing a fairly thin film so that $N$ is approximately equal to the substrate index, $N \rightarrow n_{0}$. Our design utilizes the other possibility for reducing the geometrical dispersion, which is to let $N$ become approximately equal to the film index, $N \rightarrow n_{1}$. At both limits, the geometrical dispersion vanishes [4].

\section{Experiments with Leaky Coupling}

Leaky output coupling has been employed in measuring the attenuation of low-loss light-guiding films, in the arrangements shown schematically in Fig. 9. The principle is that the light of the guided beam is coupled out of the film under test into a photodiode; diode and coupler are moved along the guided beam, and the logarithm of the detector signal is recorded as a function of the position along the guide similar as in Fig. 6. The slope of this function is the attenuation constant of the film. The advantage of this method is that the detector receives the full power of the guided light beam so that the measurement is affected neither by the irregular distribution of the scattering centers in the film nor by a (weak) systematic variation of the film thickness [26].

Figure $9 \mathrm{a}$ shows the simplest possible arrangement. The guide to be tested is of the normal, total reflection type. The high-index cladding $n_{p}$, which makes the guide leaky, is applied to the film in the form of a drop of high-index immersion oil. The drop is held by capillary action in the narrow gap $(0.1 \mathrm{~mm})$ between the film and the flat, large-area photodiode (solar cell). In this way, the leakage light is fed directly into the diode. The index of the immersion oil is chosen so that the coupling length $l_{m}$ of the mode to be tested is a small fraction, e.g. $1 / 5$ to $1 / 10$, of the length of the detector. This ensures complete transfer of the light power to the diode. When the latter is moved along the guide the oil drop follows closely.

Because minute amounts of the immersion oil usually form a trace behind the travelling diode, it must be moved in the direction opposite to the guided beam. Moreover, the use of an immersion
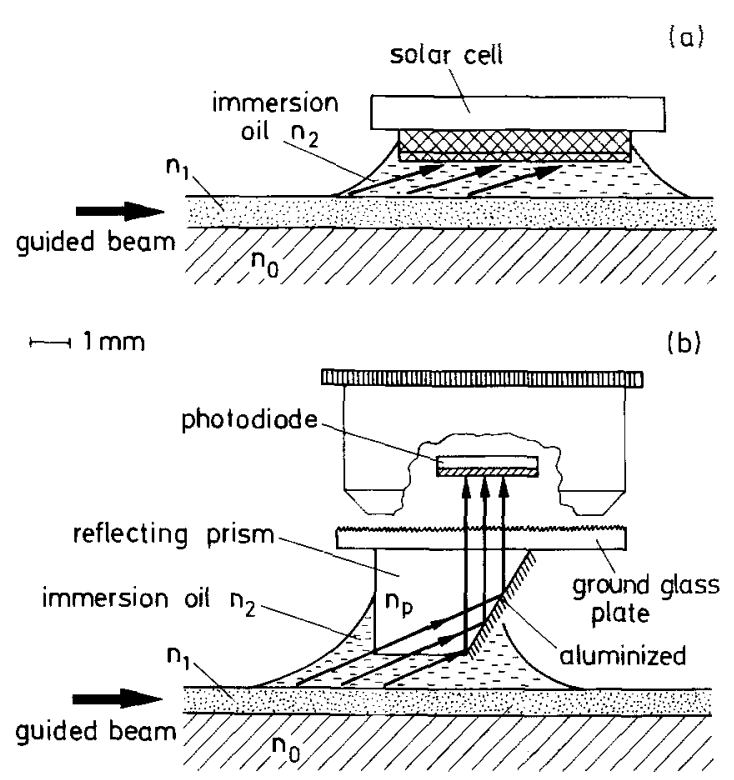

Fig. 9. Leaky wave couplers, used for measuring the attenuation of low-loss light-guides. The refractive index $n_{2}$ of the liquid must exceed the index $n$, of the film material, so that the guide becomes leaky. a) The light is coupled out of the guide into a large-area photo-detector. b) A reflecting prism of index $n_{p} \approx n_{2}$ deflects the output light onto the photodiode at nearly normal incidence. The diode is not in direct contact with the oil

liquid restricts this method of measurement to films from which the liquid can be washed off afterwards unless it is considered as a destructive measuring method.

The coupler shown in Fig. $9 \mathrm{~b}$ is basically the same as that in Fig. 9a, but it employs a photodiode of smaller area. Here, the leaky wave enters a small prism of the same (or higher) index as the oil. One face of the prism is inclined and aluminized so that it reflects the leaky wave into the photodiode. A rough-ground glass plate serves as a diffuser in case the sensitivity of the diode is spatially nonuniform. This coupler is much smaller than the one in Fig. 9a, the base of the prism being typically $1 \mathrm{~mm}$ long; such a coupler could be a useful tool in experimenting with small integrated optical circuits, in particular because it should work equally well with an optical strip-line whose surface is accessible.

\section{Summary and Conclusion}

We have discussed the guiding of light in a general slab guide, using throughout the conceptually simple picture of a plane wave that bounces in a zigzag fashion between the two surfaces of the slab, resulting 
in the average in a propagation along the guide. In the general discussion, we did not specify the nature of the claddings. Rather, we characterized them through their reflection coefficients $r_{1 j}$, which permitted us to derive general expressions for the dispersion and attenuation of a slab guide. Three factors contribute to the attenuation: the absorption $\kappa_{1}$ in the film material, and the deviations $\Delta_{j}=1$ $-\left|r_{1 j}\right|$ from total reflection at the two interfaces. From this point of view it is not necessary to differentiate between various reasons of nontotal reflections. The deviations may be the result of absorption by the cladding materials (lossy lightguide) or of refraction of the zigzagging wave into the cladding (leaky light-guide). These two mechanisms of attenuation have been illustrated by considering the operation of the normal high-index guide above and below cutoff, as well as the low-index guide. In situations where the zigzag wave travels at close to grazing incidence, the attenuation due to leakage or absorption in the substrate vanishes approximately as $(k W)^{-3}$ with increasing film thickness $W$, and the propagation of light through the slab guide tends to assume the character of free optical propagation. This $W^{-3}$ dependence has been demonstrated experimentally.

We have pointed out the significance of the "equivalent thickness" $W_{\text {eq }}$ for all questions relating to the power flow or to the absorption in the guide. The equivalent thickness is greater than the slab thickness (except in special cases) because at each reflection the zigzag wave penetrates slightly into the cladding materials (the Goos-Hänchen effect). As a consequence the "hopping length" $L$, by which the wave advances in one zigzag, is also longer.

A close relationship exists between the leaky guide discussed here and the prism-film coupler. The latter may be considered as a leaky guide whose leakage is reduced by the presence of the low-index gap. The gap can be omitted if it is technically acceptable to control the leak rate by the film thickness alone. In practice, this requires films of approx. $3 \mu \mathrm{m}$ thickness. Such a coupler is technically attractive not only because of its simplicity, but also because it can be designed for broad-band operation.

\section{References}

1. R.Shubert, J.H.Harris: IEEE Trans. MTT 16, 1048 (1968)

2. P.K.Tien: Appl. Opt. 10, 2395 (1971)

3. S.E. Miller: IEEE J. QE-8, 199 (1972)

4. R.Ulrich, R.J.Martin: Appl. Opt. 10, 2077 (1971)

5. P.K.Tien, R. Ulrich: J. Opt. Soc. Am. 60, 1325 (1970)

6. W. N. Hansen: J. Opt. Soc. Am. 58, 380 (1968) R. E.Collin: Field theory of guided waves (McGraw Hill Book Comp., New York 1960)

7. R. Ulrich: J. Opt. Soc. Am. 60, 1337 (1970)

8. T. Tamir, A.A. Oliner: Proc. IEE 10, 310 (1963)

9. K.Artmann: Ann. Physik (6) 2,87(1948). - A discussion of this relation and an extensive bibliography on the Goos-Hänchen effect are given by H.K. V.Lotsch, Dissertation, Aachen (1970); Optik 32, 116 and 189 (1970); Optik 33, 299 and 553 (1971)

10. H.K. V.Lotsch: Optik 27, 239 (1968)

11. J.J.Burke: Optical Science Center Newsletter (Tucson, Arizona) 5, 66 (1971)

12. S. Wang, M.L.Shah, J.D.Crow: IEEE J. QE-8, 212 (1972)

13. J. Kane, H. Osterberg: J. Opt. Soc. Am. 54, 347 (1964)

14. J.E. Midwinter: IEEE J. QE-7, 339 (1971)

15. M.Born, E.Wolf: Principles of optics, 3rd ed. (Pergamon, New York 1964)

16. P. K. Tien, R.J. Martin: Appl. Phys. Letters 18, 398 (1971)

17. D. Marcuse: IEEE J. QE-8, 661 (1972)

18. T. Takano, J.Hamasaki: IEEE J. QE-8, 206 (1972)

19. E. A.J. Marcatili, R.A.Schmeltzer: Bell Syst. Tech. J. 43, 1783 (1964)

20. P.K.Tien, R. Ulrich, R.J.Martin: Appl. Phys. Letters 14, 291 (1969)

21. G.Zeidler: Topical meeting on integrated optics, guided waves, materials, and devices, paper ThA5. Las Vegas, Nevada (February 1972)

22. R. Ulrich: J. Opt. Soc. Am. 61, 1467 (1971)

23. H.Osterberg, L.W.Smith: J. Opt. Soc. Am. 54, 1078 (1964)

24. F.Zernike, J.E. Midwinter: IEEE J. QE-6, 577 (1970)

25. J.E. Midwinter: IEEE J. QE-7, 345 (1971)

26. F. Zernike, E.L.Sloan, J.C. Webster, R.B. McGraw, W.L. Knecht: Topical meeting on integrated optics, guided waves, materials, and devices, paper TuA9. Las Vegas, Nevada (February 1972)

27. T.Tamir, A.A. Oliner: IRE Trans., AP-10, 51 (1962), and Proc. IEEE, 51, 317 (1963)

28. The role of leaky waves in such couplers has been pointed out by T. Tamir and H. L. Bertoni: J. Opt. Soc. Am. 61, 1397 (1971)

Dr. R. Ulrich

Dr. W. Prettl

Max-Planck-Institut für Festkörperforschung

D-7000 Stuttgart 1

Heilbronner Straße 69

Federal Republic of Germany 